\title{
MicroRNA-602 prevents the development of inflammatory bowel diseases in a microbiota-dependent manner
}

\author{
SONG ZHAO *, LEI ZHU* , WAN FENG, LU ZHANG, DAN-DAN CHEN, YU-CUI HU and HONG SHEN \\ Department of Gastroenterology, Affiliated Hospital of Nanjing University of \\ Chinese Medicine, Nanjing, Jiangsu 210029, P.R. China
}

Received July 1, 2019; Accepted March 10, 2020

DOI: $10.3892 /$ etm.2021.10808

\begin{abstract}
Inflammatory bowel diseases (IBD) are a group of chronic disorders occurring in the intestinal tract. Previous studies demonstrated that genetics and microbiota play critical roles in the pathogenesis of IBD. Discoveries of genes that may regulate the homeostasis of gut microbiota and pathogenesis of IBD have the potential to provide new therapeutic targets for IBD treatment. The results suggested that the expression level of microRNA (miR)-602 is negatively related to the development of IBD, and that miR-602 overexpression in mice may prevent inflammation and intestinal barrier injuries in dextran sulfate sodium (DSS)-induced IBD mice. It was also found that the microbiota is important for miR-602-mediated prevention of IBD, as the inhibitory effect of miR-602 was lost when the microbiota was depleted using antibiotics. Furthermore, co-housing or adoptive transfer of microbiota from miR-602 could attenuate the pathogenesis of IBD. In addition, it was demonstrated that miR-602 could target tumor necrosis factor receptor-associated factor 6 (TRAF6) in intestinal epithelial cells. Collectively, the present results suggest that miR-602 plays a protective role in DSS-induced IBD by targeting TRAF6 in a microbiota-dependent manner.
\end{abstract}

\section{Introduction}

Inflammatory bowel diseases (IBD) are immune disorders associated with chronic inflammation in the intestinal tract, and include Crohn's diseases and ulcerative colitis (1-3). Previous studies revealed that the microbiota, genetics, immune dysregulation and stress participate in the development of IBD (3-7).

Correspondence to: Dr Hong Shen, Department of Gastroenterology, Affiliated Hospital of Nanjing University of Chinese Medicine, 155 Hanzhong Road, Nanjing, Jiangsu 210029, P.R. China

E-mail: shenhongnjucm@126.com

*Contributed equally

Key words: microRNA-602, inflammatory bowel diseases, microbiota, tumor necrosis factor receptor-associated factor 6
The microbiota is thought to play a central role in the pathogenesis of IBD, and multiple strategies have been developed to treat IBD via targeting the gut flora (8-11). Furthermore, previous studies have shown that certain microbiota or gut dysbiosis can influence the outcomes of IBD, autoimmune disorders and diabetes (12-14). Moreover, segmented filamentous bacteria alone can have an important impact on the development of IBD and also regulate the immune responses in gut $(15,16)$. Another previous study showed that the ratio of Firmicutes and Bacteroidetes can influence the sensitivity of mice to IBD induction, and a lower Firmicutes/Bacteroidetes ratio appears to be related to higher susceptibility to IBD in humans and mice (17).

The regulation of gut microbiota is very complex (18). Intestinal epithelial cells (IECs) include different cell types, such as Paneth cells, goblet cells and enterocytes, which produce antimicrobial peptides and mucus, including regenerating islet-derived protein $3 \gamma$, Reg $3 \alpha$, mucin-1 and mucin-2, to regulate the constitution and homeostasis of gut microbiota $(19,20)$. Abnormal inflammatory cytokine production in the intestine can also regulate the gut microbiota directly or indirectly (21). Immune cells can secret inflammatory cytokines such as tumor necrosis factor $\alpha(\mathrm{TNF}-\alpha)$, interleukin 17 (IL-17) and IL-22 to regulate the function of IECs, thereby influencing the balance of intestinal flora $(22,23)$. Moreover, stress can also affect the homeostasis of gut microbiota, causing mice to be more sensitive to IBD (24).

MicroRNAs (miRNA/miRs) are small non-coding RNA molecules that exist in animals and plants, and play critical roles in the regulation of gene expression $(25,26)$. Previous findings revealed that miRNAs can modulate gene expression during or after transcription by targeting specific nucleotide sequences (25). Moreover, miRNAs participate in the development of different immune disorders and cancer types (26-30). The expression of miR-602 has been found to inhibit hepatitis $\mathrm{C}$ virus genotype $1 \mathrm{~b}$ infection and regulate the expression levels of the tumor suppressor genes p53, p73 and Ras association domain family 1 isoform $\mathrm{A}$ in a hepatoma cell line (31). miR-602 is also an important signaling regulator in chondrocytes (32). Tumor necrosis factor receptor-associated factor 6 (TRAF6) signaling has be found to be involved in the development of IBD $(33,34)$, and previous studies showed that miR-146a can regulate the TRAF6 signaling pathway $(34,35)$. However, it is not fully understood whether miRNA, in 
particular miR-602, plays a role in IBD pathogenesis and the regulation of gut microbiota.

The present results suggested that miR-602 is downregulated in intestinal tissues from IBD mice. Furthermore, overexpression of miR-602 may prevent the development of IBD, increase the production of proinflammatory cytokines and decrease intestinal integrity in an IBD mouse model. In addition, the inhibitory effect of miR-602 was lost when the microbiota was depleted using an antibiotic mix. It was also found that miR-602 can target the 3'-untranslated region (3'UTR) of TRAF6 and inhibit the related signaling pathways in IECs.

\section{Materials and methods}

Bioinformatics assay. The expression level of miR-602 in intestinal tissues under normal or ulcerative colitis condition was based on GSE43009 and GSE53867 as previously described (36). GSE43009 and GSE53867 datasets were downloaded from NCBI's Gene Expression Omnibus (GEO) database (http://www.ncbi.nlm.nih.gov/geo/). The read count for miR-602 was selected and used for further analysis.

Cell culture. Non-transformed rat small intestinal IEC-6 cells (Bena Culture Collection) were cultured in DMEM (cat. no. 8112040; Gibco; Thermo Fisher Scientific, Inc.) supplemented with 10\% FBS (cat. no. 10099-141; Gibco; Thermo Fisher Scientific, Inc.), $100 \mathrm{U} / \mathrm{ml}$ penicillin and $100 \mu \mathrm{g} / \mathrm{ml}$ streptomycin (Gibco; Thermo Fisher Scientific, Inc.) at $37^{\circ} \mathrm{C}$ with $5 \% \mathrm{CO}_{2}$. Coca- 2 (American Type Culture Collection), a human intestinal epithelial cell line, was cultured in DMEM/F12 (Gibco; Thermo Fisher Scientific, Inc.) containing $10 \% \mathrm{FBS}$ and $1 \%$ penicillin-streptomycin at $37^{\circ} \mathrm{C}$ with $5 \% \mathrm{CO}_{2}$.

Mice. Experiments were performed according to the Institutional Guidelines for the Care and Use of Laboratory Animals in Research and were approved by the Committee of Affiliated Hospital of Nanjing University of Chinese Medicine. Male C57BL/6 mice (age, 6-8 weeks; weight, 20 g) were purchased from the Shanghai SLAC Laboratory Animal Co., Ltd. and raised in the Affiliated Hospital of Nanjing University of Chinese Medicine. The mice were housed at a constant room temperature $\left(23 \pm 2^{\circ} \mathrm{C}\right)$ and relative humidity $(50 \pm 10 \%)$ with free access to food and water in a fixed 12-h light/dark cycle.

Humane endpoint criteria were used in all experiments involving mice, which were monitored daily: Euthanasia was performed when mice exhibited signs of distress (weight loss $\geq 25 \%$; hunched posture; diarrhea; loss of active movements), while animals with diarrhea but with weight loss $<25 \%$ received daily intraperitoneal (i.p.) injections of $1 \mathrm{ml}$ saline $(\mathrm{x} 3$, at $8 \mathrm{~h}$ intervals) to avoid dehydration (37). At the end of the experiment or for euthanasia, animals were euthanized with an overdose of pentobarbital (i.p, $500 \mathrm{mg} / \mathrm{kg}$ supplemented as needed), deep anesthetization was confirmed by loss of the pedal withdrawal reflex. In total, 50 mice were used, and no mice were euthanized or found dead over the course of the experiments.

Induction and evaluation of IBD. To induce IBD, mice were treated with drinking water containing $3 \%$ dextran sulfate sodium (DSS; MP Biomedicals LLC). DSS water was replaced every other day. There was no restriction regarding the dose of DSS solution that was provided ad libitum for 7 consecutive days, and control mice received standard drinking water throughout the 7-day experiment. The disease index of IBD consisted of the following factors: Body weight loss, stool consistency and rectal bleeding (38). Body weight loss score was assessed as followed: i) No weight loss $=0$; ii) weight loss from $1-5 \%$ compared to baseline $=1$; iii) weight loss from $6-10 \%$ compared to baseline $=2$; iv) weight loss from 11-20\% compared to baseline =3; and v) weight loss $>20 \%$ compared to baseline $=4$. Stool consistency was determined as follows: i) Well-formed pellets $=0$; ii) pasty and semi-formed stools $=2$; and iii) liquid stools $=4$. For bleeding, the following criteria were used: i) No blood $=0$; ii) positive bleeding $=2$; and iii) gross bleeding $=4$.

Adenovirus (Ad) vector treatment. Adenovirus vectors (pAdEasy/Track-CMV) used in the present study were purchased from Agilent Technologies Inc. Blank vectors without miR-602 were used as negative controls. To overexpress miR-602 (5'-GACACGGGCGACAGCUGCGGC CC-3'), $100 \mu \mathrm{l}$ of adenovirus suspension was injected intravenously into 8 -week-old mice $\left(2 \times 10^{9}\right.$ particles/g body weight) retro-orbitally using a 0.5 insulin syringe (Becton, Dickinson and Company) on day 1 and 3. The treatment lasted for 7 days, during which the mice were checked daily. After adenovirus treatment, mice were treated with $3 \%$ DSS (w/v) in drinking water for 7 days to establish an IBD mouse model.

Fecal microbiota depletion and fecal microbiota transplantation (FMT). For fecal microbiota depletion, antibiotics were administered in autoclaved drinking water for 4 weeks in the following concentrations: Vancomycin $(1 \mathrm{~g} / \mathrm{l})$, ampicilin $(1.5 \mathrm{~g} / \mathrm{l})$, kanamycin $(1 \mathrm{~g} / \mathrm{l})$ and metronidazole $(1.5 \mathrm{~g} / \mathrm{l})$. This time course is consistent with standard antibiotic administration used in multiple studies for altering microbiota (39). After microbiota depletion, mice were treated with $3 \%$ DSS (w/v) in drinking water for 7 days to establish the IBD mouse model.

For mice with microbiota depletion, FMT was performed as described previously (40). In the morning, feces were collected and immediately diluted (1:20 w/vol) in sterile, anoxic Ringer buffer (Beijing Solarbio Science \& Technology, Co., Ltd.) containing $1 \mathrm{~g} / 1$ L-cysteine (Sigma-Aldrich; Merck KGaA) as a reducing agent. This was used as inoculum. Colonization was achieved by intragastric gavage with $200 \mu \mathrm{l}$ inoculum once per day for 5 consecutive days. A total of 10 days after FMT, mice were treated with DSS for 7 days to establish the IBD mice model.

The mice were housed at a constant room temperature $\left(23 \pm 2^{\circ} \mathrm{C}\right)$ and relative humidity $(50 \pm 10 \%)$ under a fixed 12 -h light/dark cycle. They were given free access to food and water. Each cage housed a total of 5 mice. To investigate the potential role of microbiota in miR-602 mediated therapy in IBD, mice injected with adenovirus carrying negative control (Ad-NC) were co-housed with mice injected with Ad-miR-602 for three weeks, after which the IBD model was induced. A total of 5 mice were included in each group.

Fecal microbiota was isolated from Ad-NC and Ad-miR-602 mice, and these fecal microbiotas were separately transferred 
Table I. Primer used for reverse transcription-quantitative PCR.

\begin{tabular}{lll}
\hline Gene & \multicolumn{1}{c}{ Forward $\left(5^{\prime}-3 '\right)$} & \multicolumn{1}{c}{ Reverse $\left(5^{\prime}-3^{\prime}\right)$} \\
\hline TNF $\alpha(\mathrm{m})$ & ACTGAACTTCGGGGTGATCG & GTTTGCTACGACGTGGGCTA \\
$\mathrm{IL}-1 \beta(\mathrm{m})$ & TGCCACCTTTTGACAGTGATG & ATGTGCTGCTGCGAGATTTG \\
$\mathrm{IL}-6(\mathrm{~m})$ & GTCCTTCCTACCCCAATTTCCA & TAACGCACTAGGTTTGCCGA \\
TGF- $\beta(\mathrm{m})$ & GTGGACACTCGATCGCTACC & GATGCGAGGGACTCAAGAGG \\
OCLN $(\mathrm{m})$ & TTTCAGGTGAATGGGTCACCG & GCTCCCAAGATAAGCGAACCT \\
MUC-2 $(\mathrm{m})$ & TTGTCACCTTCGATGGGCTC & TCTCGTGGCGCACAATAAGT \\
MUC-3 $(\mathrm{m})$ & CAAGAAGAGCGCAAAGCAGG & CCTCCATCCCACACACTTCC \\
ZO-1 $(\mathrm{m})$ & AGAAAAAGAATGCACAGAGTTGT & GAAATCGTGCTGATGTGCCA \\
TRAF6 $(\mathrm{m})$ & TCCAGAGTTTGCCGTCCAAG & TGGGTCCCTTCAGAAGTTCA \\
GAPDH $(\mathrm{m})$ & TCTTTTGCGTCGCCAGCC & CCATGGGTGGAATCATATTGGAAC \\
TRAF6 $(\mathrm{h})$ & GCGCACTAGAACGAGCAAG & GGCAGTTCCACCCACACTAT \\
GAPDH $(\mathrm{h})$ & CTTTTGCGTCGCCAGGTGAAG & ACCAAATCCGTTGACTCCGAC \\
miR-602 $(\mathrm{h} / \mathrm{m})$ & GACACGGGCGACAGCUG & CTCGCTTCGGCAGCACA \\
U6 $(\mathrm{h} / \mathrm{m})$ & CTCGCTTCGGCAGCACA & AACGCTTCACGAATTTGCGT
\end{tabular}

m, mouse; h, human; TNF $\alpha$, tumor necrosis factor $\alpha$; IL, interleukin; TGF- $\beta$, transforming growth factor $\beta$; OCLN, occludin; MUC, Mucin; ZO-1, zonula occludens; TRAF6, Tumor necrosis factor receptor-associated factor 6.

into antibiotic-treated mice. After 10 days, IBD model was induced in these microbiota-transferred mice and the pathogenesis index was recorded.

Histological analysis. Hematoxylin and eosin (H\&E) staining was used to assess the induction of IBD. After euthanizing the mice, colon tissues from control mice and IBD mice were collected and fixed at room temperature in PBS containing $4 \%$ paraformaldehyde for $72 \mathrm{~h}$. The samples were sectioned to $6 \mu \mathrm{m}$ thickness using a microtome (cat. no. SM2500; Leica Microsystems $\mathrm{GmbH}$ ). Then, the sections were stained with hematoxylin and eosin (hematoxylin, $1.0 \mathrm{~kg} / \mathrm{l}$; eosin, $0.827 \mathrm{~kg} / \mathrm{l}$ ), and examined under light microscopy (Olympus Corporation; magnification, x100).

$R N A$ isolation and reverse transcription-quantitative $P C R$ $(R T-q P C R)$. To isolate RNA from colon tissues, a mortar and a pestle was used to grind the frozen tissues which were pre-cooled by liquid nitrogen. After euthanizing the mice, the colon tissue samples were collected. After length measurement and weighing, the tissue sample was put into liquid nitrogen and grinded. The treated samples were lysed with TRIzol ${ }^{\circledR}$ (Invitrogen; Thermo Fisher Scientific, Inc.) for extraction of mRNA. The generation of cDNA from the extracted mRNA samples was performed using the PrimeScript ${ }^{\mathrm{TM}}$ RT Reagent kit (cat. no. RR037B, Takara Bio, Inc.). After mixing RNA with the kit components as described in the user manual, the mixture was incubated at $37^{\circ} \mathrm{C}$ for $15 \mathrm{~min}$ three times, and then incubated at $85^{\circ} \mathrm{C}$ for $5 \mathrm{sec}$ to inactivate the reverse transcriptase. RT-qPCR analysis was performed to assess gene expression with Premix Ex Taq ${ }^{\mathrm{TM}}$ (Probe qPCR; cat. no. RR390W; Takara Bio, Inc.). The thermocycling conditions were as follows: $15 \mathrm{~min}$ at $95^{\circ} \mathrm{C}$ (initial denaturation), followed by 35 cycles of $15 \mathrm{sec}$ at $94^{\circ} \mathrm{C}$ (denaturation), $30 \mathrm{sec}$ at $60^{\circ} \mathrm{C}$ (annealing) and $30 \mathrm{sec}$ at $72^{\circ} \mathrm{C}$ (extension). For gene expression in cells, IEC-6 and caco- 2 cells were collected, total RNA was isolated with TRIzol ${ }^{\circledR}$ (Invitrogen; Thermo Fisher Scientific, Inc.), and reverse transcription and qPCR was performed as aforementioned. miRNA and mRNA expression levels were quantified using the $2^{-\Delta \Delta \mathrm{Cq}}$ method (41). The primers used for PCR are listed in Table I.

Western blotting. Cells were transfected with Ad-NC and Ad-miR-602 using Lipofectamine ${ }^{\circledR} 2000$ (Thermo Fisher Scientific, Inc.). After $24 \mathrm{~h}$ incubation at $37^{\circ} \mathrm{C}$, cell samples were collected and lysed with Cell lysis buffer (Beyotime Institute of Biotechnology) containing the protease inhibitor cocktail (Beyotime Institute of Biotechnology) for western blotting and IP. After the protein concentration was determined using a bicinchoninic acid protein assay kit (Beyotime Institute of Biotechnology), protein extracts $(10 \mu \mathrm{g})$ were analyzed via $12 \%$ SDS-PAGE. The proteins were then transferred onto nitrocellulose membranes, which were subsequently incubated with the following primary antibodies at $4^{\circ} \mathrm{C}$ overnight: TRAF6 (1:1,000; cat. no. ab227560; Abcam) and GAPDH (1:2,000; cat. no. 60004; ProteinTech Group, Inc.). After washing with PBST in triplicate, horseradish peroxidase-conjugated anti-anti-rabbit (1:2,000; cat. no. SA00001-7L; ProteinTech Group, Inc.) or anti-mouse (1:2,000; cat. no. SA00001-1; ProteinTech Group, Inc.) secondary antibodies were added and incubated at room temperature for $2 \mathrm{~h}$. The membranes were then washed with Tris-buffered saline and $0.1 \%$ Tween-20 in triplicate. Protein levels were assessed using an enhanced chemiluminescence mix (Thermo Fisher Scientific, Inc.).

Luciferase reporter assay. The luciferase reporter assay was performed as described previously (42). MODE-K and Caco-2 cells were co-infected with wild-type or mutant TRAF6-3'UTR-Luc firefly luciferase constructs (100 ng) and $40 \mathrm{nmol} / \mathrm{l} \mathrm{miR-602} \mathrm{mimics} \mathrm{using} \mathrm{Lipofectamine} 2000$ reagent 
A

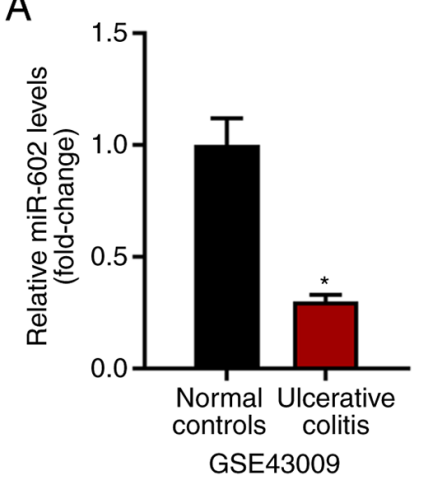

B

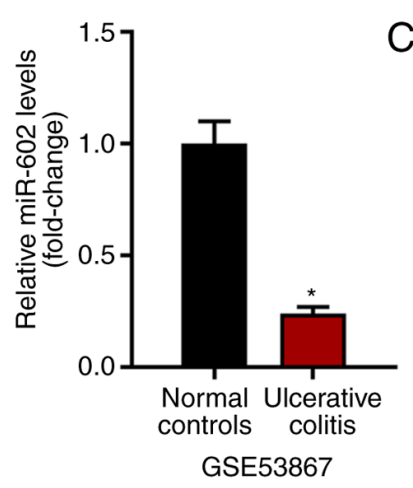

C

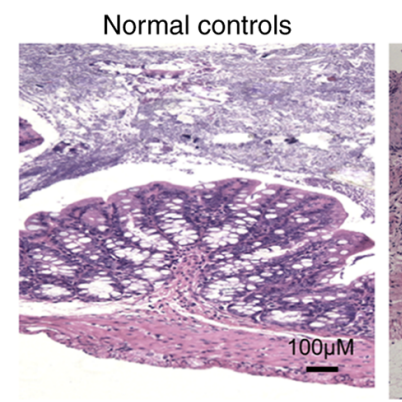

DSS colitis

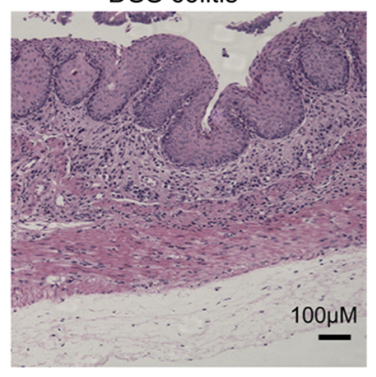

D

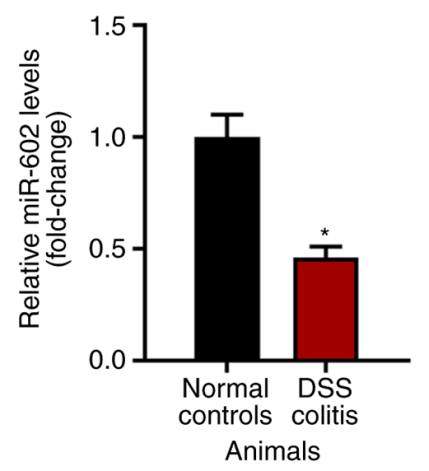

Figure 1. Expression of miR-602 is downregulated in the intestinal tissues of DSS-induced IBD mice. Analysis of the miR-602 expression in the (A) GSE43009 and (B) GSE53867. (C) Histological analysis of intestinal tissues in healthy mice and DSS-induced IBD mice. (D) Expression levels of miR-602 in the intestinal tissues from healthy mice and IBD mice $(n=5-6)$. $\mathrm{P}<0.05$ vs. healthy control group. IBD, inflammatory bowel diseases; miR, microRNA; DSS, dextran sulfate sodium.

(Thermo Fisher Scientific, Inc.). Then, 2 ng of pRL-SV40 plasmids (Addgene, Inc.) were infected to assess the transfection efficiency. After incubation for $24 \mathrm{~h}$ at $22^{\circ} \mathrm{C}$, luciferase activity was analyzed using the Dual-Luciferase ${ }^{\circledR}$ Reporter $\left(D_{L}{ }^{\mathrm{TM}}\right)$ Assay System (Promega Corporation).

Statistical analysis. All experiments were performed $\geq 3$ times. Data are presented as the mean \pm SEM. Data were analyzed using a one-way ANOVA with Tukey's multiple comparison test or an unpaired two-tailed Student's t-test. $\mathrm{P}<0.05$ was considered to indicate a statistically significant difference.

\section{Results}

Expression level of miR-602 is negatively related to IBD development. The present study investigated the expression level of miR-602 in intestinal tissues under normal or ulcerative colitis conditions based on GSE43009 and GSE53867. It was found that, compared with normal intestinal tissues from healthy mice, tissues with ulcerative colitis had a decreased expression level of miR-602 (Fig. 1A and B). To examine this result, IBD was induced using DSS (Fig. 1C) and the intestinal tissues were collected for RNA analysis. It was found that intestinal tissues from IBD mice had decreased miR-602 expression compared with intestinal tissue from control mice (Fig. 1D). Collectively, the present results suggested that the expression level of miR-602 is negatively related to IBD development.
Overexpression of miR-602 alleviates IBD in mice. To investigate the role of miR-602 in IBD development, miR-602 was overexpressed in mice by injecting Ad-miR-602. After 5 days, it was found that the expression level of miR-602 was increased in intestinal tissues in mice with Ad-miR-602 compared with those injected with Ad-NC (Fig. 2A). The present study established an IBD mouse model using DSS, and then recorded body weight, occult blood, and diarrhea score. The present results suggested that overexpression of miR-602 increased body weight, but significantly decreased occult blood, diarrhea score and disease activity index (Fig. 2C-E). Compared with healthy mice, Ad-NC treated mice showed a significantly higher colonic weight/length ratio, while the colonic weight/length ratio of mice overexpressing miR-602 was similar to healthy mice (Fig. 2F). Therefore, the present results indicated that miR-602 may alleviate IBD.

Furthermore, the mRNA expression level of inflammatory cytokines and the genes related to intestinal integrity were investigated. It was found that miR-602 overexpression significantly inhibited mRNA expression levels of the proinflammatory cytokines TNF- $\alpha$, IL-1 $\beta$ and IL- 6 , but increased the expression levels of the anti-inflammatory cytokine transforming growth factor $\beta$ (TGF- $\beta$; Fig. $2 \mathrm{G}$ ). Moreover, miR-602 overexpression promoted the expression of occludin, zonula occludens 1 (ZO-1), Mucin 2 (MUC-2) and MUC-3 (Fig. 2H), indicating that miR-602 may prevent against the destruction of intestinal tissue integrity. Collectively, the present results suggested that miR-602 could have a protective role in IBD. 
A
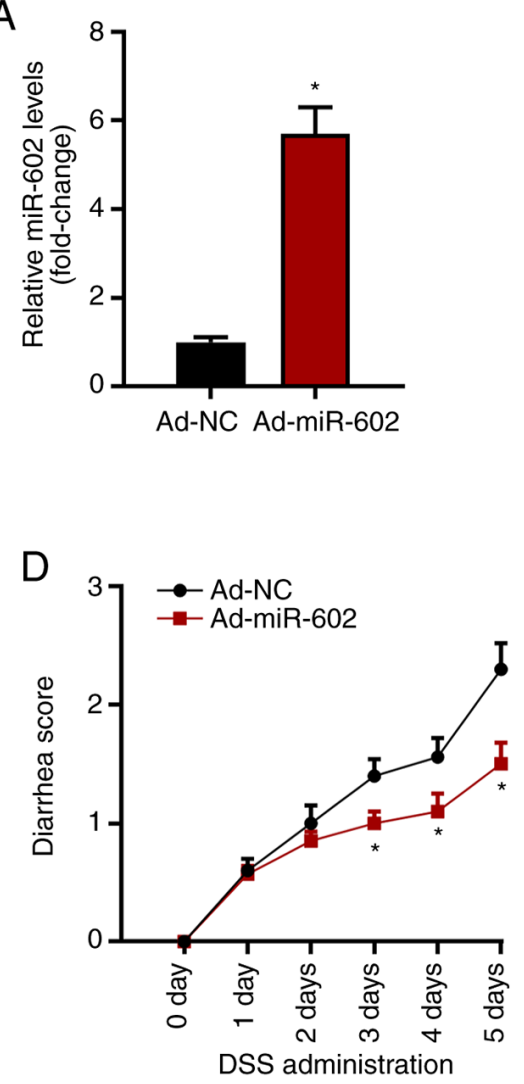

B

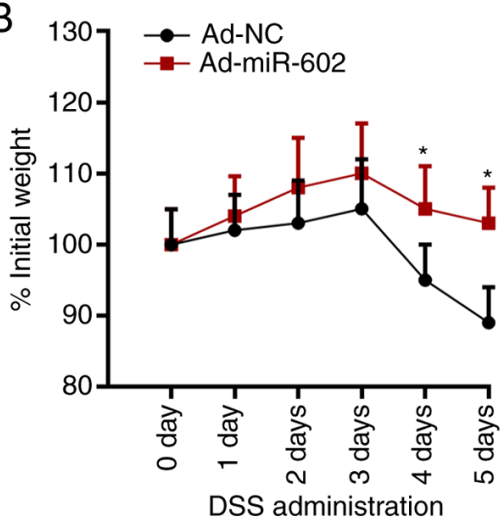

$\mathrm{E}$

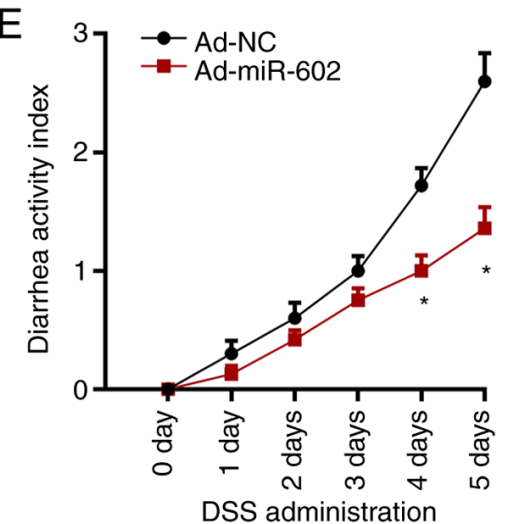

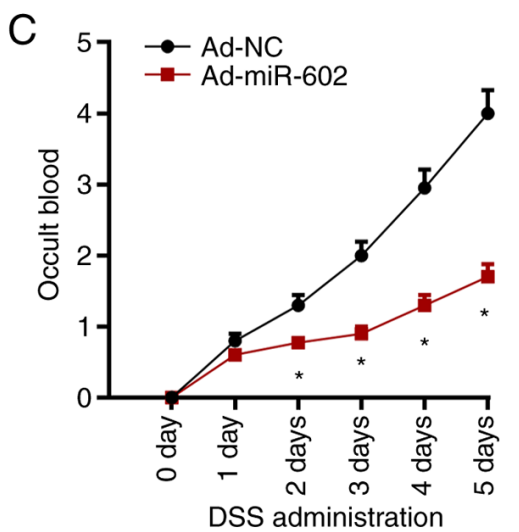

F

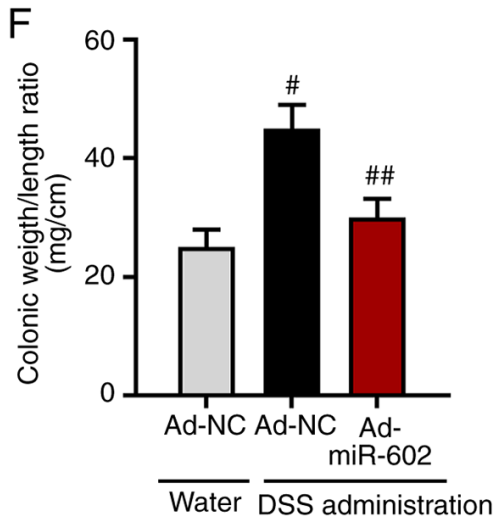

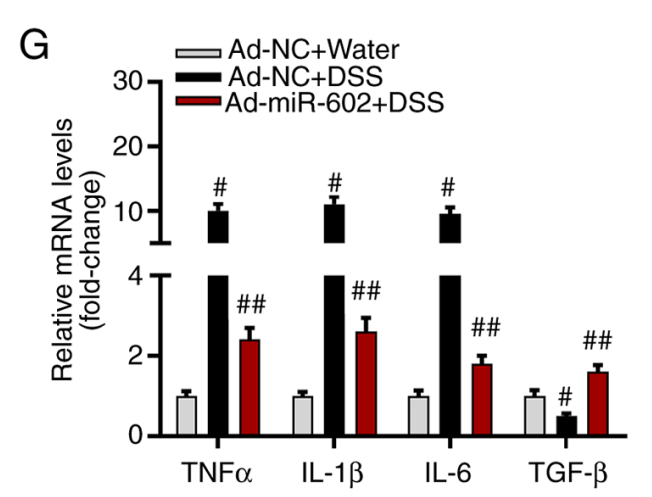

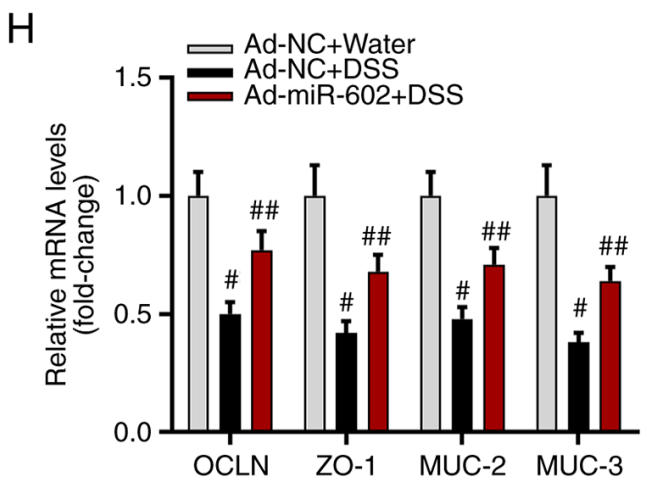

Figure 2. Overexpression of miR-602 alleviates IBD. Mice were injected with Ad-NC or Ad-miR-602. (A) Expression level of miR-602. Mice transfected with Ad-NC or Ad-miR-602 were treated with DSS to induce IBD. * $\mathrm{P}<0.05$ vs. the Ad-NC group. (B) Body weight, (C) occult blood, (D) diarrhea score and (E) diseases activity index were analyzed. All, " $\mathrm{P}<0.05$ vs. the Ad-NC group. (F) Colonic weight and length were measured, and the ratio of weight to length was calculated. ${ }^{\#} \mathrm{P}<0.05$ and ${ }^{\# \#} \mathrm{P}<0.01$ vs. the Ad-NC water group. mRNA expression levels of (G) TNF- $\alpha$, IL-1 $\beta$, IL- 6 and TGF- $\beta$. (H) mRNA expression levels of OCLN, ZO-1, MUC-2 and MUC-3. n=5-6. " $\mathrm{P}<0.05$ and ${ }^{\# \#} \mathrm{P}<0.01$ vs. the corresponding Ad-NC water group. TNF $\alpha$, tumor necrosis factor $\alpha$; IL, interleukin; TGF- $\beta$, transforming growth factor $\beta$; OCLN, occludin; MUC, Mucin; ZO-1, zonula occludens; TRAF6, Tumor necrosis factor receptor-associated factor 6; IBD, inflammatory bowel diseases; miR, microRNA; DSS, dextran sulfate sodium; Ad, adenovirus; NC, negative control.

Co-housing healthy, miR-602-overexpressing mice with IBD mice showed beneficial effects on IBD. Microbiota has been shown to play critical roles in the development of IBD (43). To investigate the potential role of microbiota in miR-602 mediated therapy in IBD, mice injected with Ad-NC were co-housed with mice injected with Ad-miR-602, after which IBD was induced 5 days later. An IBD model was also induced in Ad-NC mice without co-housing, which were used as control. It was found that those in the Ad-NC + Ad-miR-602 group had little difference in body weight change compared with the Ad-NC + Ad-NC group (Fig. 3A). Furthermore, the Ad-NC + Ad-miR-602 group exhibited less severity in occult blood, diarrhea and disease scores (Fig. 3B-D). It was also demonstrated that, while co-housing with Ad-miR-602 mice did not significantly influence the colonic weight/length ratio, co-housing decreased the expression levels of TNF- $\alpha$ and increased the expression of the cellular integrity-related gene ZO-1 in intestinal tissues. Mice could transmit microbes to others upon transient co-housing through coprophagy and grooming behaviors. Therefore, the present results suggested that microbiota from miR-602-overexpressing mice may be transferred to Ad-NC mice during the co-housing process, and that the microbiota plays a protective role in miR-602-mediated IBD. 

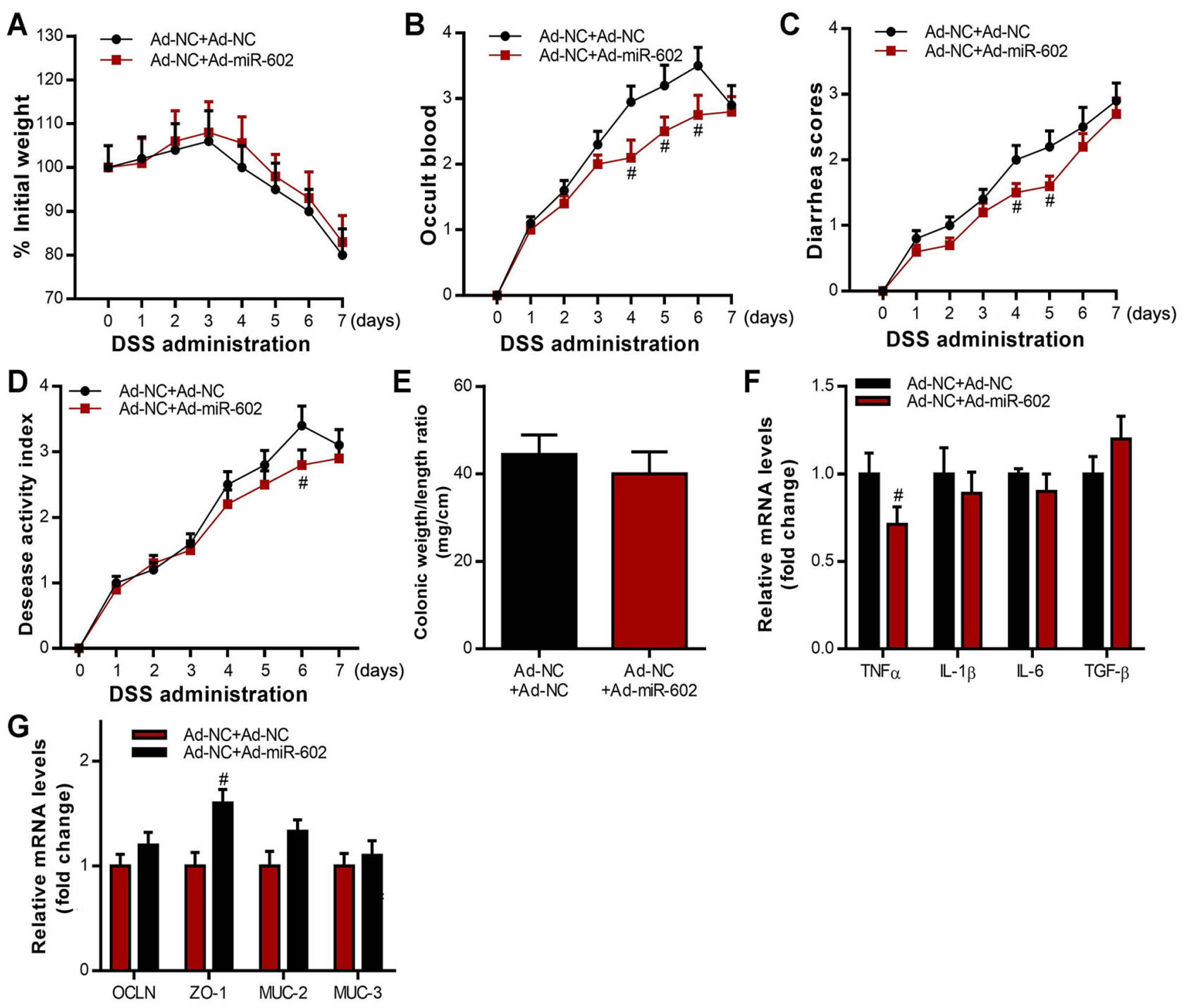

Figure 3. Co-housing Ad-miR-602-treated mice attenuates IBD in Ad-NC-treated mice. Ad-NC-treated mice were co-housed with or without Ad-miR-602treated mice, then induced with IBD. (A) Body weight, (B) occult blood, (C) diarrhea score and (D) diseases activity index of mice were analyzed. (E) Colonic weight and length were recorded, and the ratio of weight to length was calculated. (F) mRNA expression levels of TNF- $\alpha$, IL-1 $\beta$, IL- 6 and TGF- $\beta$. (G) mRNA expression levels of OCLN, ZO-1, MUC-2 and MUC-3. TNF $\alpha$, tumor necrosis factor $\alpha$; IL, interleukin; TGF- $\beta$, transforming growth factor $\beta$; OCLN, occludin; MUC, Mucin; ZO-1, zonula occludens-1; TRAF6, Tumor necrosis factor receptor-associated factor 6; IBD, inflammatory bowel diseases; miR, microRNA; DSS, dextran sulfate sodium; Ad, adenovirus; NC, negative control. $n=5-6$. ${ }^{\#} \mathrm{P}<0.05$ vs. the Ad-NC+Ad-NC group in B-D, F, and G.

Overexpression of miR-602 alleviates IBD in a microbiotadependent manner. To further investigate whether miR-602 alleviates IBD via the microbiota, antibiotics were used to deplete the gut microbiota in Ad-NC mice and Ad-miR-602 mice, and their responses to DSS-induced IBD were assessed. The results revealed that the inhibitory effect of miR-602 on body weight, occult blood, diarrhea score, disease activity index and colonic weight/length ratio was lost when gut microbiota were depleted (Fig. 4A-E). As presented in Fig. 4F, the inhibitory effect of miR-602 on the expression of various pro-inflammatory cytokines, including TNF- $\alpha$, IL-1 $\beta$, IL-6 and TNF- $\beta$ was also lost when gut microbiota were depleted. In contrast, the stimulatory effect of miR-602 on the expression of cellular integrity-related genes, including OCLN, ZO-1, MUC-2 and MUC-3, was inhibited when gut microbiota were depleted (Fig. 4G). These results suggested that gut microbiota depletion abrogated the effects of miR-602 overexpression by alleviating IBD and regulating gene expression in the colon.
Fecal microbiota was isolated from Ad-NC and Ad-miR-602 mice, and these fecal microbiota were separately transferred into antibiotic-treated mice. After 10 days, IBD was induced in the microbiota-transferred mice and the pathogenesis was recorded. The present results indicated that compared to Ad-NC-FMT mice, those that received fecal microbiota from Ad-miR-602 mice had a higher expression level of miR-602 in the colon (Fig. 5A), thus suggesting a bi-directional interaction between miR-602 and gut microbiota. Furthermore, fecal microbiota from Ad-miR-602 mice, as opposed to that from Ad-NC mice, prevented weight loss, occult blood and diarrhea in IBD mice (Fig. 5B-E). It was found that Ad-miR-602 mouse-derived fecal microbiota also reduced the colonic weight/length ratio (Fig. 5F). The expression levels of inflammatory cytokines and genes related to intestinal integrity were also examined, and it was demonstrated that Ad-miR-602 mouse-derived fecal microbiota decreased the expression levels of the proinflammatory genes TNF- $\alpha$, IL-1 $\beta$ and IL-6 (Fig. 5G). 

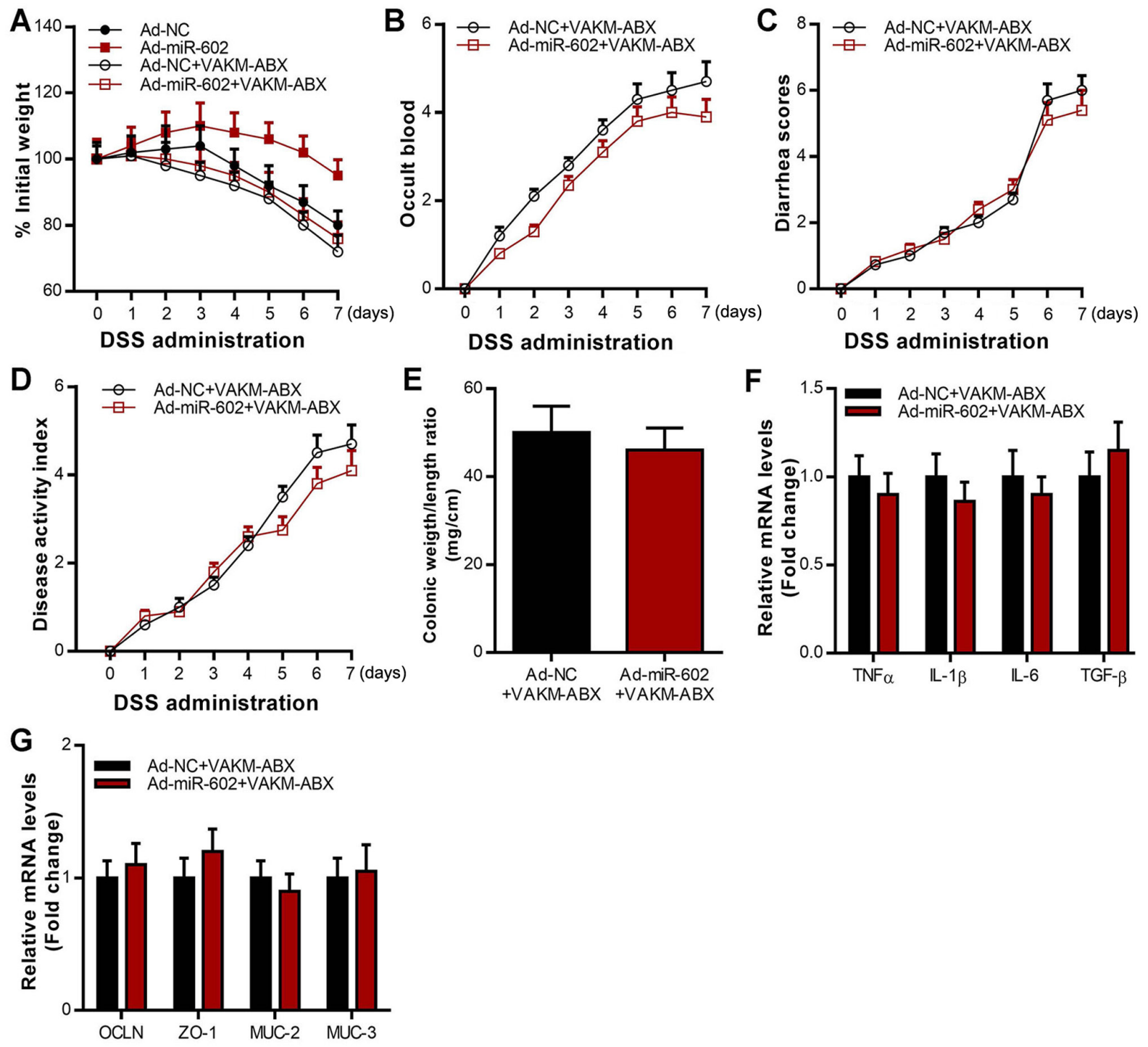

Figure 4. Antibiotics treatment abrogates the beneficial effects of miR-602 overexpression in IBD mice. Mice were treated with Ad-NC or Ad-miR-602, and then the gut microbiota was depleted using an antibiotics mix. Then, DSS was used to induce IBD. (A) Body weight, (B) occult blood, (C) diarrhea score and (D) disease activity index. (E) Colonic weight and length were measured, and the ratio of weight to length was calculated. (F) mRNA expression of TNF- $\alpha$, IL-1 $\beta$, IL-6, TGF- $\beta$. (G) mRNA expression level of OCLN, ZO-1, MUC-2 and MUC-3. n=5-6. TNF $\alpha$, tumor necrosis factor $\alpha$; IL, interleukin; TGF- $\beta$, transforming growth factor $\beta$; OCLN, occludin; MUC, Mucin; ZO-1, zonula occludens-1; TRAF6, Tumor necrosis factor receptor-associated factor 6; IBD, inflammatory bowel diseases; miR, microRNA; DSS, dextran sulfate sodium; Ad, adenovirus; NC, negative control; VAKM, the combination of vancomycin (1 g/l), ampicilin (1.5 g/l), kanamycin (1 g/l), and metronidazole (1.5 g/l); ABX, antibiotics. $\mathrm{n}=5-6$.

Moreover, Ad-miR-602 mouse-derived fecal microbiota significantly increased the expression level of TGF- $\beta$ (Fig. 5G) and intestinal integrity-related genes (Fig. 5H). Collectively, the present results suggested that miR-602 may help to prevent the development of IBD in a microbiota-dependent manner.

miR-602 inhibits TRAF6 signaling in intestinal tissues. The present study investigated whether miR-602 regulates IBD via the TRAF6 signaling pathway in intestinal tissues. It was demonstrated that TRAF6 expression level is significantly increased in IBD mice compared with healthy control mice (Fig. 6A). Moreover, overexpression of miR-602 significantly inhibited TRAF6 mRNA and protein expression levels in IEC-6 and Caco-2 cells (Fig. 6B-D). Furthermore, using a luciferase reporter assay it was found that the mutation in 3'UTR miR-602 binding sites of TRAF6 (Fig. 6E) abrogated the effects of wild-type miR-602 on TRAF6 expression level (Fig. 6F and G).

\section{Discussion}

Genetic mutations and microenvironment exposure are the most important factors for the occurrence of IBD $(4,7)$, however, recent studies have demonstrated that the microbiota also plays a critical role in the progression of autoimmune disorders, such as colitis $(10,11,44)$. Thus, targeting microbiota or developing microbiota-related medicine may be an effective strategy to facilitate the treatment of IBD. However, it is not fully understood how the constitution and homeostasis of gut microbiota is regulated.

Previous studies have shown that miRNAs play an important role in the initiation and development of IBD (28). It has 

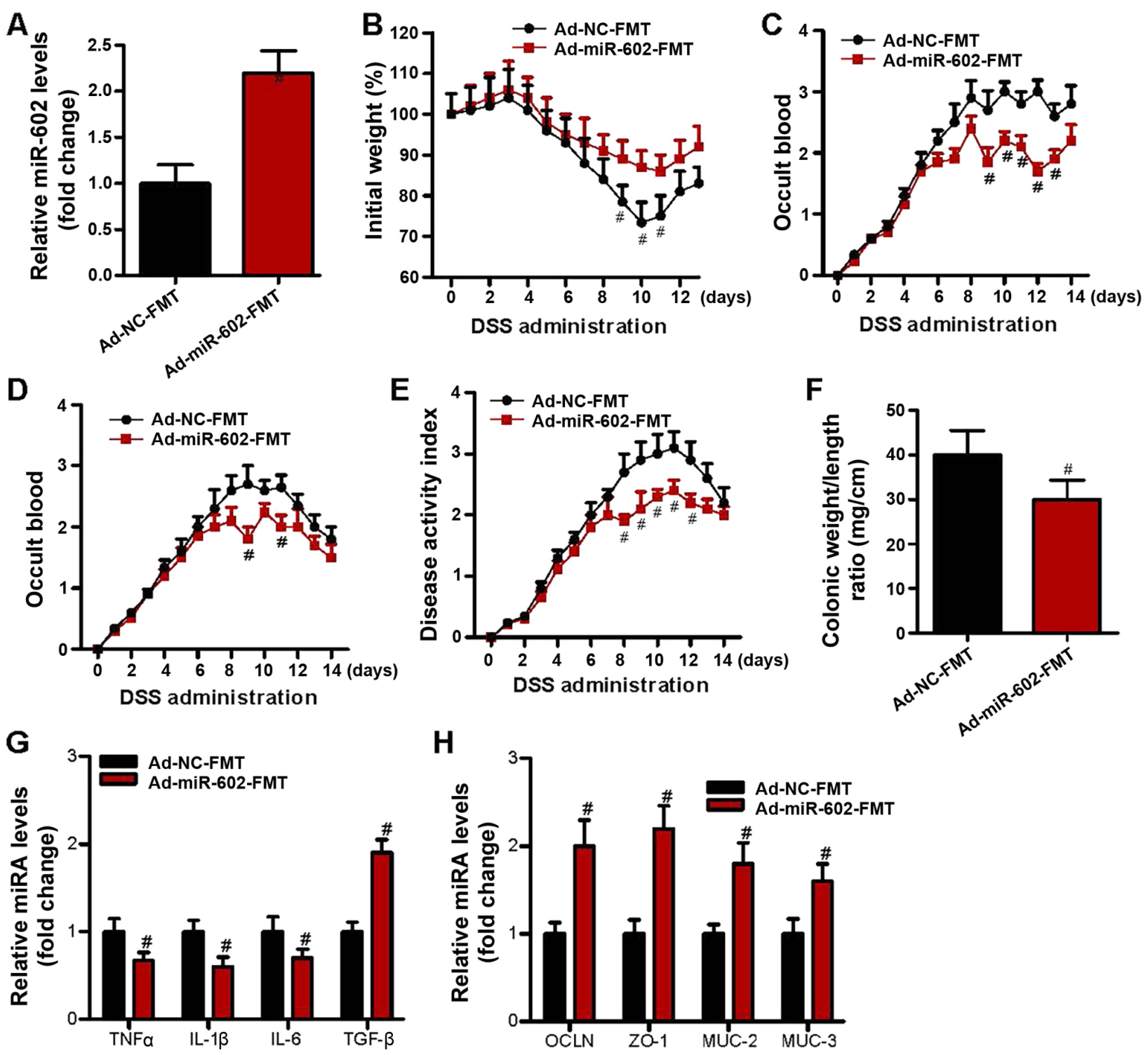

Figure 5. Fecal microbiota from Ad-miR-602-treated mice attenuated IBD in mice. Fecal microbiota was isolated from the Ad-NC or Ad-miR-602-treated mice derived feces, and were transferred into antibiotic-treated mice. DSS containing drinking water was used to induce IBD. (A) Expression level of miR-602 in Ad-NC-FMT and Ad-miR-602-FMT groups. (B) Body weight, (C) occult blood, (D) diarrhea score and (E) disease activity index of the mice. (F) Colonic weight and length were used to calculate the ratio of weight to length. (G) mRNA expression levels of TNF- $\alpha$, IL-1 $\beta$, IL- 6 and TGF- $\beta$. (H) mRNA expression levels of OCLN, ZO-1, MUC-2 and MUC-3. FMT, fecal microbiota transplantation; TNF $\alpha$, tumor necrosis factor $\alpha$; IL, interleukin; TGF- $\beta$, transforming growth factor $\beta$; OCLN, occludin; MUC, Mucin; ZO-1, zonula occludens-1; TRAF6, Tumor necrosis factor receptor-associated factor 6; IBD, inflammatory bowel diseases; miR, microRNA; DSS, dextran sulfate sodium; Ad, adenovirus; NC, negative control. $\mathrm{n}=6 .{ }^{*} \mathrm{P}<0.05$ vs. the Ad-NC-FMT group.

been previously shown that miR-148a is downregulated in human IBD and in tissues of patient with colorectal cancer, and that restoration of miR-148a expression inhibits colitis and colitis-associated tumorigenesis in mice (45). By contrast, miR-155 is increased in the colonic mucosa and peripheral blood in patients with IBD, suggesting that miR-155 may act as a biomarker for the diagnosis of IBD (46). Moreover, a recent study showed that the microbiota decreases the expression level of host miR-665, which then increases the expression level of ATP Binding Cassette Subfamily C Member 3 in the host (46). In contrast to these previous studies, the present results suggested that miR-602 regulated the microbiota, which then alleviated IBD development. In addition, the present study found that the regulation of gut flora by miR-602 may be achieved via the targeting of TRAF6 in IECs, which play critical roles in the regulation of gut microbiota (47). Therefore, further studies on the exact mechanisms of miR-602 on IECs may facilitate the development of a pharmacological target for the prevention and treatment of IBD.

Abnormal production of inflammatory cytokines is considered to be related to the development of colitis $(48,49)$, however, neutralization of inflammatory cytokines does not always result in the alleviation of colitis (50), suggesting that the dysregulation of inflammatory cytokines may be caused by other upstream events (50). The present results suggested that miR-602 downregulated the production of certain proinflammatory cytokines, while upregulating the production of the anti-inflammatory cytokine TGF- $\beta$. Furthermore, these effects were dependent on the gut microbiota, as overexpression of miR-602 was not seen to have inhibitory effects in 


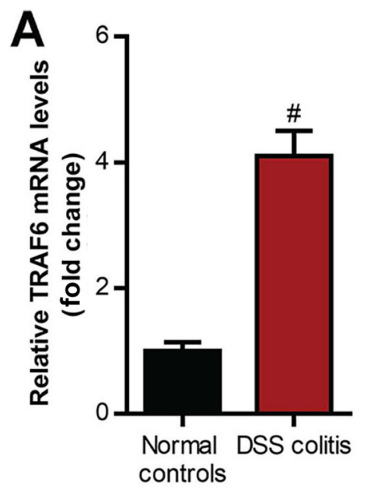

D
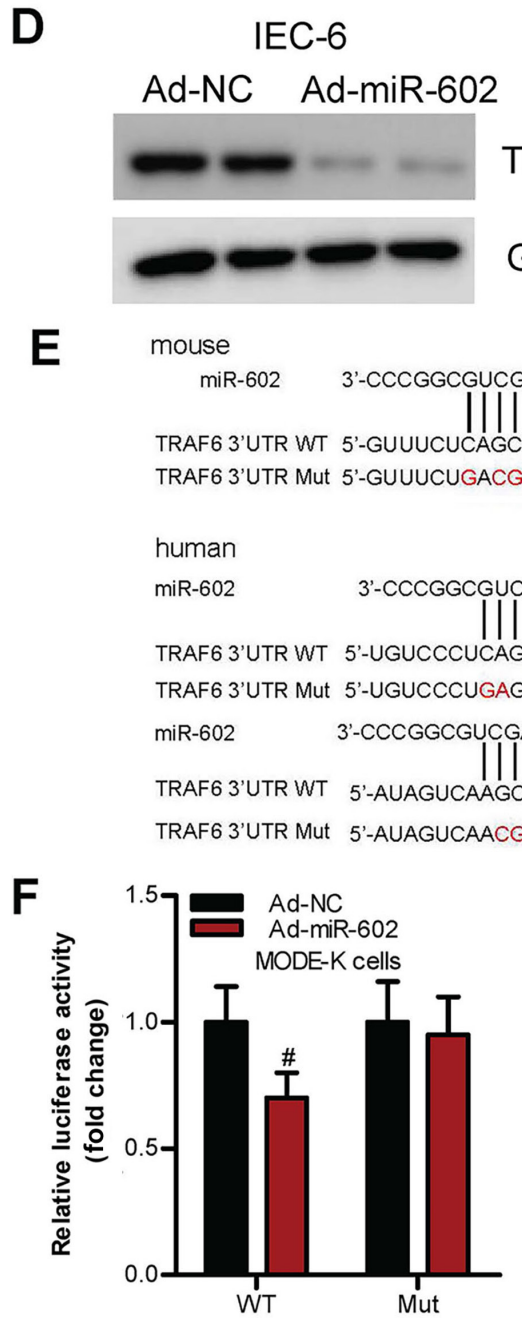
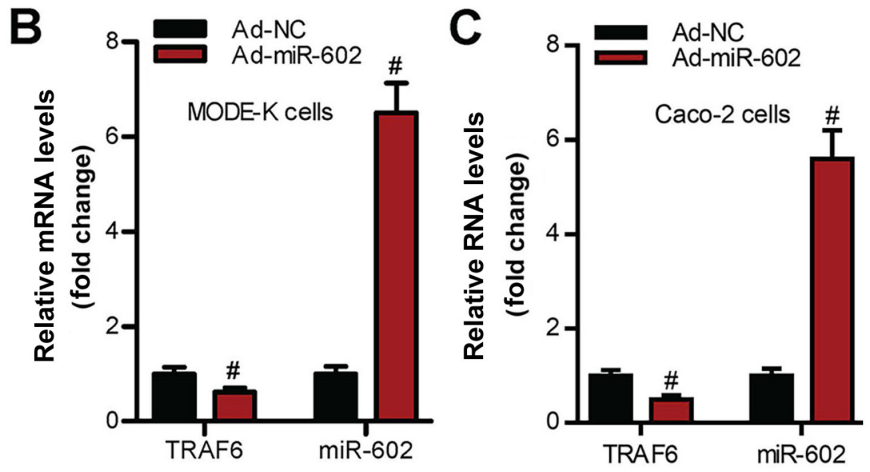

Caco-2

TRAF6

GAPDH
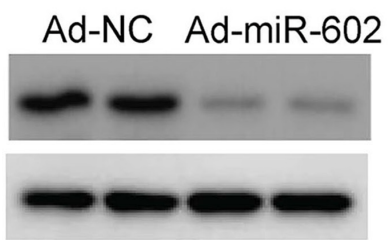

UCGACAGCGGGCACAG-5'

\|\|$\|$

AGCCAGCCAG-3'

$$
\text { IIII\|GCAGCGGGCACAG-5' }
$$

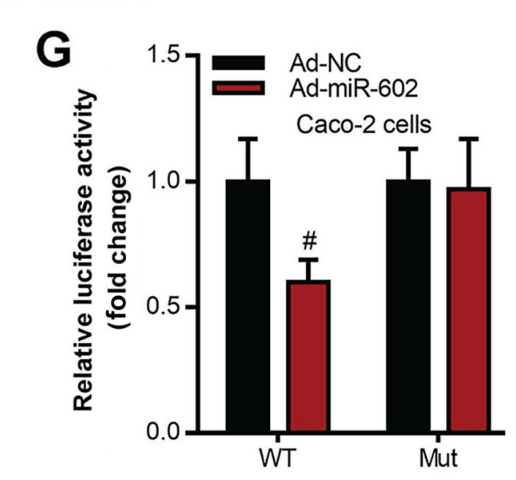

Figure 6. miR-602 targets TRAF6 signaling in IEC. (A) Expression level of TRAF6 in intestinal tissues from healthy mice and IBD mice. mRNA expression levels of TRAF6 and miR-602 in (B) IEC-6 and (C) Caco-2 cells treated with Ad-NC or Ad-miR-602. (D) Protein expression levels of TRAF6 in IEC-6 and Caco-2 cells treated with Ad-NC or Ad-miR-602. (E) TRAF6 3'UTR Mut site of mouse and human. There are two binding sites in the TRAF6 3'UTR, both of the binding sites were mutated. WT and TRAF6 3'UTR Mut in (F) Mode-K and (G) Caco-2 cell lines treated with Ad-NC or Ad-miR-602. The expression of TRAF6 was calculated by testing the luciferase activities. TRAF6, Tumor necrosis factor receptor-associated factor 6; IBD, inflammatory bowel diseases; miR, microRNA; DSS, dextran sulfate sodium; Ad, adenovirus; NC, negative control; WT, wild-type; Mut, mutant; 3'UTR, 3'untranslated regions. n=5-6. ${ }^{\#} \mathrm{P}<0.05$ vs. Ad-NC group.

microbiota-depleted mice. Thus, dysbiosis in the gut may cause hyperactivation of the immune system, and therapies targeting microbiota may be an effective method to control immune responses during colitis.

Adoptive transfer of microbiota or the use of microbial preparation are considered to be ideal strategies for the treatment of autoimmune disorders and cancer types $(51,52)$. The present study found that miR-602 may shape the gut microbiota balance, even under a co-housing situation. Furthermore, the present results suggested that overexpression of miR-602 exerted beneficial effects on IBD mice, indicating this miR-602 may regulate the constitution and colonization of gut microbiota. Moreover, microbiota has been found to influence the development of different kinds of immune disorders, such as experimental autoimmune encephalomyelitis and cancer types $(53,54)$. Thus, future studies could investigate the combination of microbiota 
adoptive transfer and miR-602 overexpression, which may be a potential therapeutic method for colitis.

Furthermore, the present study identified that miR-602 exerted its inflammatory effect via the inhibition of TRAF6 expression. Previous studies have shown that TRAF6 plays a critical role in IBD (33). It has been shown that TRAF6 neddylation drives inflammatory arthritis by increasing $\mathrm{NF}-\kappa \mathrm{B}$ activation, which then activate the expression of various inflammatory cytokines including TNF- $\alpha$, IL- 6 and IL-8 (45).

Collectively, the present results suggested that miR-602 may alleviate IBD in a microbiota-dependent manner and that IECs play important roles in such processes. The present results provide further evidence on the regulation of the gut microbiota and facilitate the development of strategies for the treatment of IBD.

\section{Acknowledgements}

Not applicable.

\section{Funding}

This project was sponsored by the Program of The State Administration of Traditional Chinese Medicine of China (grant no. JDZX2015083) and the National Natural Science Foundation of China (grant no. 81774243).

\section{Availability of data and materials}

The datasets used and/or analyzed during the current study are available from the corresponding author on reasonable request.

\section{Authors' contributions}

HS contributed to the design and conception of the study and revised it carefully for important intellectual content. SZ, LeZ, WF, DDC and LuZ acquired the data by screening papers identified on PubMed. DDC and YCH revised the study critically for important intellectual content. SZ, LeZ, WF and $\mathrm{YCH}$ were involved in drafting the manuscript. SZ and $\mathrm{YCH}$ analyzed and interpreted the data. SZ also contributed to the conception and design of the study and revised the manuscript. All authors agreed to be accountable for all aspects of the work in ensuring that questions related to the accuracy or integrity of any part of the work are appropriately investigated and resolved. All authors read and approved the final manuscript.

\section{Ethics approval and consent to participate}

This study was approved by the Committee of Affiliated Hospital of Nanjing University of Chinese Medicine.

\section{Patient consent for publication}

Not applicable.

\section{Competing interests}

The authors declare that they have no competing interests.

\section{References}

1. Hodson R: Inflammatory bowel disease. Nature 540: S97, 2016.

2. Chu H, Khosravi A, Kusumawardhani IP, Kwon AH, Vasconcelos AC, Cunha LD, Mayer AE, Shen Y, Wu WL, Kambal A, et al: Gene-microbiota interactions contribute to the pathogenesis of inflammatory bowel disease. Science 352: 1116-1120, 2016.

3. Neurath MF: Cytokines in inflammatory bowel disease. Nat Rev Immunol 14: 329-342, 2014.

4. Kaser A, Zeissig S and Blumberg RS: Inflammatory bowel disease. Annu Rev Immunol 28: 573-621, 2010.

5. Maloy KJ and Powrie F: Intestinal homeostasis and its breakdown in inflammatory bowel disease. Nature 474: 298-306, 2011.

6. Cho JH: The genetics and immunopathogenesis of inflammatory bowel disease. Nat Rev Immunol 8: 458-466, 2008.

7. Khor B, Gardet A and Xavier RJ: Genetics and pathogenesis of inflammatory bowel disease. Nature 474: 307-317, 2011.

8. Liang J, Sha SM and Wu KC: Role of the intestinal microbiota and fecal transplantation in inflammatory bowel diseases. J Dig Dis 15: 641-646, 2014.

9. Lopetuso LR, Petito V, Graziani C, Schiavoni E, Paroni Sterbini F, Poscia A, Gaetani E, Franceschi F, Cammarota G, Sanguinetti M, et al: Gut microbiota in health, diverticular disease, irritable bowel syndrome, and inflammatory bowel diseases: Time for microbial marker of gastrointestinal disorders. Dig Dis 36: 56-65, 2018.

10. Wehkamp J and Frick JS: Microbiome and chronic inflammatory bowel diseases. J Mol Med (Berl) 95: 21-28, 2017.

11. Miyoshi J and Chang EB: The gut microbiota and inflammatory bowel diseases. Transl Res 179: 38-48, 2017.

12. Baumgart DC and Carding SR: Inflammatory bowel disease: Cause and immunobiology. Lancet 369: 1627-1640, 2007.

13. Campbell AW: Autoimmunity and the gut. Autoimmune Dis 2014: 152428, 2014.

14. Musso G, Gambino R and Cassader M: Obesity, diabetes, and gut microbiota: The hygiene hypothesis expanded? Diabetes Care 33: 2277-2284, 2010.

15. Yi J, Jung J, Han D, Surh CD and Lee YJ: Segmented filamentous bacteria induce divergent populations of Antigen-Specific CD4 T cells in the small intestine. Mol Cells 42: 228-236, 2019.

16. Ivanov II, Atarashi K, Manel N, Brodie EL, Shima T, Karaoz U, Wei D, Goldfarb KC, Santee CA, Lynch SV, et al: Induction of intestinal Th17 cells by segmented filamentous bacteria. Cell 139: 485-498, 2009.

17. Molist F, Manzanilla EG, Perez JF and Nyachoti CM: Coarse, but not finely ground, dietary fibre increases intestinal Firmicutes:Bacteroidetes ratio and reduces diarrhoea induced by experimental infection in piglets. Br J Nutr 108: 9-15, 2012.

18. Boulangé CL, Neves AL, Chilloux J, Nicholson JK and Dumas ME: Impact of the gut microbiota on inflammation, obesity, and metabolic disease. Genome Med 8: 42, 2016.

19. Liu X, Lu J, Liu Z, Zhao J, Sun H, Wu N, Liu H, Liu W, Hu Z, Meng G, et al: Intestinal Epithelial Cell-Derived LKB1 suppresses colitogenic microbiota. J Immunol 200: 1889-1900, 2018.

20. Sekirov I, Russell SL, Antunes LC and Finlay BB: Gut microbiota in health and disease. Physiol Rev 90: 859-904, 2010.

21. Ding Y, Yanagi K, Cheng C, Alaniz RC, Lee K and Jayaraman A: Interactions between gut microbiota and non-alcoholic liver disease: The role of microbiota-derived metabolites. Pharmacol Res 141: 521-529, 2019.

22. Ramirez-Carrozzi V, Sambandam A, Luis E, Lin Z, Jeet S, Lesch J, Hackney J, Kim J, Zhou M, Lai J, et al: IL-17C regulates the innate immune function of epithelial cells in an autocrine manner. Nat Immunol 12: 1159-1166, 2011.

23. Munoz M,Eidenschenk C, Ota N, Wong K, Lohmann U, Kühl AA, Wang X, Manzanillo P, Li Y, Rutz S, et al: Interleukin-22 induces interleukin-18 expression from epithelial cells during intestinal infection. Immunity 42: 321-331, 2015.

24. Gao X, Cao Q, Cheng Y, Zhao D, Wang Z, Yang H, Wu Q, You L, Wang Y, Lin Y, et al: Chronic stress promotes colitis by disturbing the gut microbiota and triggering immune system response. Proc Natl Acad Sci USA 115: E2960-E2969, 2018.

25. Mehta A and Baltimore D: MicroRNAs as regulatory elements in immune system logic. Nat Rev Immunol 16: 279-294, 2016.

26. Li $\mathrm{Z}$ and Rana TM: Therapeutic targeting of microRNAs: Current status and future challenges. Nat Rev Drug Discov 13: 622-638, 2014. 
27. Whiteoak SR, Felwick R, Sanchez-Elsner $\mathrm{T}$ and Fraser Cummings JR: MicroRNAs in inflammatory bowel diseases: Paradoxes and possibilities. Inflamm Bowel Dis 21: 1160-1165, 2015.

28. Kalla R, Ventham NT, Kennedy NA, Quintana JF, Nimmo ER, Buck AH and Satsangi J: MicroRNAs: New players in IBD. Gut 64: 504-517, 2015.

29. Wu F, Zikusoka M, Trindade A, Dassopoulos T, Harris ML, Bayless TM, Brant SR, Chakravarti S and Kwon JH: MicroRNAs are differentially expressed in ulcerative colitis and alter expression of macrophage inflammatory peptide-2 alpha. Gastroenterology 135: 1624-1635.e24, 2008.

30. Neudecker V, Haneklaus M, Jensen O, Khailova L, Masterson JC, Tye H, Biette K, Jedlicka P, Brodsky KS, Gerich ME, et al: Myeloid-derived miR-223 regulates intestinal inflammation via repression of the NLRP3 inflammasome. J Exp Med 214: 1737-1752, 2017

31. Yang L, Ma Z, Wang D, Zhao W, Chen L and Wang G: MicroRNA-602 regulating tumor suppressive gene RASSF1A is overexpressed in hepatitis B virus-infected liver and hepatocellular carcinoma. Cancer Biol Ther 9: 803-808, 2010.

32. Akhtar N, Makki MS and Haqqi TM: MicroRNA-602 and microRNA-608 regulate sonic hedgehog expression via target sites in the coding region in human chondrocytes. Arthritis Rheumatol 67: 423-434, 2015.

33. Shen J, Qiao Y, Ran Z and Wang T: Different activation of TRAF4 and TRAF6 in inflammatory bowel disease. Mediators Inflamm 2013: 647936, 2013.

34. Wu H, Fan H, Shou Z, Xu M, Chen Q, Ai C, Dong Y, Liu Y, Nan Z, Wang Y, et al: Extracellular vesicles containing miR-146a attenuate experimental colitis by targeting TRAF6 and IRAK1. Int Immunopharmacol 68: 204-212, 2019.

35. Stickel N, Prinz G, Pfeifer D, Hasselblatt P, Schmitt-Graeff A, Follo M, Thimme R, Finke J, Duyster J, Salzer U and Zeiser R: miR-146a regulates the TRAF6/TNF-axis in donor T cells during GVHD. Blood 124: 2586-2595, 2014.

36. Xia Z, Huang L, Yin P, Liu F, Liu Y, Zhang Z, Lin J, Zou W and Li C: L-Arginine alleviates heat stress-induced intestinal epithelial barrier damage by promoting expression of tight junction proteins via the AMPK pathway. Mol Biol Rep 46: 6435-6451, 2019.

37. Chen Z, Yu K, Zhu F and Gorczynski R: Over-Expression of CD200 protects mice from dextran sodium sulfate induced colitis. PLoS One 11: e0146681, 2016.

38. Zaki MH, Boyd KL, Vogel P, Kastan MB, Lamkanfi M and Kanneganti TD: The NLRP3 inflammasome protects against loss of epithelial integrity and mortality during experimental colitis. Immunity 32: 379-391, 2010.

39. Rodrigues RR, Greer RL, Dong X, Dsouza KN, Gurung M, Wu JY, Morgun A and Shulzhenko N: Antibiotic-Induced alterations in gut microbiota are associated with changes in glucose metabolism in healthy mice. Front Microbiol 8: 2306, 2017.

40. Le Roy T, Debédat J, Marquet F, Da-Cunha C, Ichou F, Guerre-Millo M, Kapel N, Aron-Wisnewsky J and Clément K Comparative evaluation of microbiota engraftment following fecal microbiota transfer in mice models: Age, kinetic and microbial status matter. Front Microbiol 9: 3289, 2019.
41. Livak KJ and Schmittgen TD: Analysis of relative gene expression data using real-time quantitative PCR and the 2(-Delta Delta C(T)) Method. Methods 25: 402-408, 2001.

42. Yu X, Wang D, Wang X, Sun S, Zhang Y, Wang S, Miao R, Xu X and Qu X: CXCL12/CXCR4 promotes inflammation-driven colorectal cancer progression through activation of RhoA signaling by sponging miR-133a-3p. J Exp Clin Cancer Res 38: $32,2019$.

43. Khan I, Ullah N, Zha L, Bai Y, Khan A, Zhao T, Che T and Zhang C: Alteration of gut microbiota in Inflammatory Bowel Disease (IBD): Cause or consequence? IBD treatment targeting the gut microbiome. Pathogens 8: 126, 2019.

44. Dalal SR and Chang EB: The microbial basis of inflammatory bowel diseases. J Clin Invest 124: 4190-4196, 2014.

45. Zhu Y, Gu L, Li Y,Lin X, Shen H, Cui K, Chen L, Zhou F, Zhao Q Zhang J, et al: miR-148a inhibits colitis and colitis-associated tumorigenesis in mice. Cell Death Differ 24: 2199-2209, 2017.

46. Ye YL, Pang Z, Gu W and Zheng JJ: Expression of microRNA-155 in inflammatory bowel disease and its clinical significance. Zhonghua Yi Xue Za Zhi 97: 3716-3719, 2017 (In Chinese).

47. Han D, Walsh MC, Cejas PJ, Dang NN, Kim YF, Kim J, Charrier-Hisamuddin L, Chau L, Zhang Q, Bittinger K, et al: Dendritic cell expression of the signaling molecule TRAF6 is critical for gut microbiota-dependent immune tolerance. Immunity 38: 1211-1222, 2013.

48. Zundler S and Neurath MF: Pathogenic T cell subsets in allergic and chronic inflammatory bowel disorders. Immunol Rev 278: 263-276, 2017.

49. Strober W and Fuss IJ: Proinflammatory cytokines in the pathogenesis of inflammatory bowel diseases. Gastroenterology 140: 1756-1767, 2011.

50. Perrier $\mathrm{C}$ and Rutgeerts P: Cytokine blockade in inflammatory bowel diseases. Immunotherapy 3: 1341-1352, 2011.

51. Roy S and Trinchieri G: Microbiota: A key orchestrator of cancer therapy. Nat Rev Cancer 17: 271-285, 2017.

52. Zhu W, Winter MG, Byndloss MX, Spiga L, Duerkop BA, Hughes ER, Büttner L, de Lima Romão E, Behrendt CL, Lopez CA, et al: Precision editing of the gut microbiota ameliorates colitis. Nature 553: 208-211, 2018.

53. Chu F, Shi M, Lang Y, Shen D, Jin T, Zhu J and Cui L: Gut microbiota in multiple sclerosis and experimental autoimmune encephalomyelitis: Current applications and future perspectives. Mediators Inflamm 2018: 8168717, 2018.

54. Vivarelli S, Salemi R, Candido S, Falzone L, Santagati M, Stefani S, Torino F, Banna GL, Tonini G and Libra M: Gut microbiota and cancer: From pathogenesis to therapy. Cancers (Basel) 11: 38, 2019. International (CC BY-NC-ND 4.0) License. 